\title{
Full blood counts are not predictive of the risk of medication-related osteonecrosis of the jaws: a case- control study
}

\author{
Kelly McGowan, BOH (Dent Sci), Grad Dip Dent, ${ }^{\mathrm{a}, \mathrm{b}}$ Robert S Ware, BSc (Hons I) Statistics, PhD, \\ Caroline Acton, BDSc, MDSc (Oral Surg), FRACDS (OMS), ${ }^{\mathrm{d}}$ \\ Saso Ivanovski, BDSc, BDentSt, MDSc (Perio), PhD, FICD, FADI, ${ }^{\mathrm{b}}$ and \\ Newell W Johnson, MDSc, PhD, FDSRCS, FRACDS, FRCPath, FFOPRCPA, FMedSci ${ }^{\text {a,c,e }}$
}

Objectives. The role of immune function in susceptibility to medication-related osteonecrosis of the jaws (MRONJ) remains unclear. This study investigated whether full blood counts, as a measure of systemic health and immune function, predict the development of MRONJ.

Study Design. A case-control study was conducted in Brisbane, Australia. A total of 57 cases diagnosed with MRONJ from January 2010 to March 2017 were identified from hospital records and individually matched with up to 4 controls using primary disease, sex, age, and antiresorptive therapy (total sample =249). Demographic and clinical data were extracted and associations were investigated using conditional logistic regression.

Results. A total of $67 \%$ of cases and $65 \%$ of controls reported at least 1 result outside of the laboratory reference range (odds ratio $=0.7 ; 95 \%$ confidence interval: $0.3,1.5 ; P=.29)$. The most commonly reported abnormal results were low hemoglobin (53\% of cases, $48 \%$ of controls) and low hematocrit (33\% of cases, $25 \%$ of controls). There were no significant differences between groups in any of the reported parameters.

Conclusions. Patients taking antiresorptive medications often return blood test results outside the standard laboratory reference range. Altered blood counts were not limited to patients who developed MRONJ and do not appear to be clinically useful in identifying patients at high risk for this condition. (Oral Surg Oral Med Oral Pathol Oral Radiol 2019;000:1-8)

Suppression and dysfunction of the natural immune response has been implicated in the development of medication-related osteonecrosis of the jaws (MRONJ). ${ }^{1,2}$ It is widely reported that higher doses of antiresorptives (ARs), an extended duration of AR therapy, and dental extractions are associated with an increased risk of this rare complication. ${ }^{1,2}$ However, not every patient who develops MRONJ will report these risk factors ${ }^{3}$ and not all patients with these risk factors will develop MRONJ. ${ }^{4}$ Other patient-specific factors appear to play a critical role in determining susceptibility, and immune dysfunction is a possible candidate.

The innate and adaptive immune systems may both play a role in the pathogenesis of MRONJ.

\footnotetext{
K. McGowan has received a speaker honorarium from Eli Lilly. ${ }^{\text {a S}}$ School of Dentistry and Oral Health, Griffith University, Gold Coast, Queensland, Australia.

${ }^{\mathrm{b}}$ School of Dentistry, University of Queensland, Herston, Queensland, Australia.

${ }^{c}$ Menzies Health Institute Queensland, Griffith University, Gold Coast, Queensland, Australia.

${ }^{\mathrm{d} O r a l} \&$ Maxillofacial Surgery Department, Royal Brisbane and Women's Hospital, Herston, Queensland, Australia.

${ }^{\mathrm{e}}$ Faculty of Dentistry, Oral and Craniofacial Sciences, King's College, London, UK.

Received for publication Feb 16, 2019; returned for revision Apr 1, 2019; accepted for publication Apr 8, 2019.

(C) 2019 Elsevier Inc. All rights reserved.

2212-4403/\$-see front matter

https://doi.org/10.1016/j.oooo.2019.04.004
}

Chemotherapy and prolonged corticosteroids have been associated with an increased risk of MRONJ. ${ }^{5}$ Multiple myeloma patients, who are primarily immunosuppressed as a result of their aberrant plasma cells but are further compromised by the corticosteroids and chemotherapies used to treat their cancer, are reported to experience the highest rate of MRONJ. ${ }^{6}$ There is in vivo and in vitro evidence to suggest bisphosphonates (BPs) are taken up by neutrophils, ${ }^{7}$ resulting in decreased survival and function of this cell population. ${ }^{8}$ In addition, clinical results indicate that nitrogen-containing BPs decrease subsets of innate T cells, ${ }^{9}$ and patients who developed MRONJ lacked immune resiliency and normal function. ${ }^{10}$ Macrophages also appear to be susceptible to medications associated with MRONJ, with evidence to suggest they are inhibited by denosumab, BPs, and antiangiogenics. ${ }^{11,12}$ Given that medical and dental histories of patients who develop MRONJ vary significantly, it seems likely that immune function would be one of a number of causal

\section{Statement of Clinical Relevance}

Patients who take antiresorptive medications often return blood tests with 1 or more parameters outside the standard laboratory reference range, but this does not correlate with their risk of developing medication-related osteonecrosis of the jaws and cannot be used to identify high-risk patients. 
components in a multifactorial disease pathway in MRONJ. The high microbial load in the mouth and the relative ease with which routine function and dental procedures can breach the integrity of the oral mucosa are likely to compound the effects of impaired immunity in a small subset of patients and result in MRONJ.

Most clinical research concerning laboratory parameters associated with MRONJ has focused on C-terminal telopeptide tests, which have been subsequently been found not to correlate with the risk of developing MRONJ. ${ }^{13,14}$ Minimal data are available on the complete blood cell counts of patients who develop MRONJ, and consequently it is difficult to determine the role of systemic immune function in susceptibility to MRONJ. Leukopenia $^{15}$ and anaemia ${ }^{5,16}$ have previously been reported as possible risk factors, and lower C-reactive protein (CRP) levels have been associated with successful treatment of MRONJ. ${ }^{17}$ White blood cell counts (WBCCs) were found to not be associated with treatment outcomes, ${ }^{18,19}$ but leukopenia has been identified as an important prognostic factor for recurrence. ${ }^{20} \mathrm{No}$ significant difference was found in the laboratory parameters of patients with osteoradionecrosis and MRONJ, ${ }^{21}$ but this was not referenced to a control group. It was hypothesized that patients with an impaired immune response and/or results outside of the laboratory reference range would be more likely to develop MRONJ. The aim of this study was to investigate whether common laboratory test results, as markers of systemic health and immune function, are associated with an increased risk of developing MRONJ.

\section{METHODS}

\section{Study design}

A case-control study was conducted in South East Queensland, Australia, to investigate the association between standard laboratory blood test results and the development of MRONJ. Patients treated at the Royal Brisbane \& Women's Hospital (RBWH) and the Gold Coast University Hospital (GCUH) between January 1, 2010 and March 31, 2017 were eligible for inclusion. Both RBWH and GCUH are tertiary level specialist hospitals with a specialist oral and maxillofacial surgery department. Ethical approval for the study was obtained from the RBWH Human Research Ethics Committee and Griffith University Human Research Ethics Committee. The reporting of this study conforms to the Strengthening the Reporting of Observational Studies in Epidemiology statement. ${ }^{22}$

\section{Case selection}

Cases were identified by searching RBWH and GCUH patient databases using the International Classification of Disease and Related Health Problems (ICD-10-AM 9th Edition) code K10.2 ("Inflammatory conditions of the jaw"). Medical records were manually reviewed to confirm a clinical diagnosis of MRONJ according to the American Association of Oral and Maxillofacial Surgeon's 2014 diagnostic criteria. ${ }^{1}$ Patients were eligible for inclusion if they were aged older than 18 years, had previously taken antiresorptive or antiangiogenic medications, presented with exposed bone in the oral cavity that did not heal within 8 weeks, and had no history of radiation therapy or metastatic disease of the jaw. Patients were excluded if they did not have a full blood count result available within the 3-12 months before their MRONJ diagnosis, or if they were pregnant or breastfeeding. Blood tests within 3 months of a patient's MRONJ diagnosis were excluded to reduce the risk of reverse causality where early inflammation or infection associated with the onset of MRONJ caused alterations in systemic markers.

\section{Control selection}

Controls with the same primary disease were identified from the same treating hospital using ICD-10 codes for multiple myeloma, breast cancer, prostate cancer, osteoporosis, rheumatoid arthritis, and Paget's disease. Up to 4 controls were individually matched to each case on age, sex, primary disease, and AR therapy. Sex and age (to within 7 years) were matched to eliminate potential confounding from age-related factors and hormonal variations. The dose and duration of AR medication are reported to increase the risk of MRONJ, ${ }^{1}$ and therefore controls with higher doses and/or longer treatment histories were selected where exact matching was not possible. Controls were excluded if the results of a full blood count were not available within the 12 months before their index date.

\section{Variables and data collection}

The outcome variable was the presence or absence of clinically diagnosed MRONJ and explanatory variables were the laboratory parameters ascertained from blood tests. Pathology results were provided by the Queensland Health Clinical Information Systems Support Unit, and the data managers who extracted the blood test results were masked to the case/control status of the patients. Because this study aimed to determine whether full blood count results predict MRONJ, it was important to include blood tests that were collected and analyzed before a clinical diagnosis of MRONJ. The diagnostic criteria for MRONJ specifies lesions that have not healed within 8 weeks, ${ }^{1}$ and therefore blood tests at least 3 months but not more than 1 year before MRONJ diagnosis were selected for cases. This was intended to exclude changes in systemic markers that resulted from the inflammatory and infective processes associated with developing or established MRONJ. The most recently available blood tests 
before index date were used for controls. From the full blood count, red blood cell count, WBCC, hematocrit, hemoglobin, mean corpuscular hemoglobin, mean corpuscular volume, and mean platelet volume were recorded. Neutrophil, lymphocyte, monocyte, eosinophil, and basophil counts and percentages were also recorded. Blood test results came from 4 different laboratories, and reference ranges varied slightly between laboratories for some parameters. For this reason, results for each category were recorded as within or outside the laboratory-specific reference range. Fasting blood glucose, free thyroxine, thyroid-stimulating hormone, vitamin D, CRP, erythrocyte sedimentation rate, and full blood counts were recorded where available.

Demographic information, history of antiresorptive therapy, and comorbidities and medications were recorded from hospital records. Diabetes, thyroid disease, chemotherapy, corticosteroids, kidney disease, tobacco use, and autoimmune disease were considered possible confounders. The patient's first dose of AR medication was determined from chemotherapy medication orders, hospital medical action plans, or outpatient medication lists. The Australian Bureau of Statistics Index of Relative Socio-Economic Disadvantage and Advantage ${ }^{23}$ was used to calculate socioeconomic status at the postcode level, and this was categorized into thirds based on Queensland rankings.

\section{Statistical methods}

Sample size calculations were based on the assumption of a binary exposure variable (categorized as within or outside the laboratory reference range). We assumed 60
MRONJ cases would be identified, with an average of 3 matched controls per case. Prior data indicated the average probability of a control returning a blood test result outside of the laboratory reference range was 0.5 . With $80 \%$ power and $\alpha=.05$ we would be able to detect a significant difference where the odds ratio (OR) was $\geq 2.2$.

Statistics were summarized as frequencies and the $\chi^{2}$ test was used to compare the clinical and demographic characteristics of cases and controls. Conditional logistic regression determined the unadjusted OR and $95 \%$ confidence intervals (CI) where the exposure was laboratory test results and the outcome was MRONJ. Models were conditioned on their individually matched case-control groups. Adjusted ORs were calculated using multivariable conditional logistic regression. Smoking, kidney disease, and duration of AR therapy were included as potentially confounding variables because these factors differed significantly between groups in the univariable analysis. A prespecified stratified analysis of oncologic and nononcologic subgroups was conducted. Statistical analyses were conducted using IBM SPSS Statistics, Version 25 (IBM Corp., Armonk, NY, USA).

\section{RESULTS}

Fifty-seven confirmed cases of MRONJ had blood test results available in the 3-12 months before diagnosis. It was not possible to match 4 controls to each case, and on 36 occasions 3 controls were matched (192 controls overall) to each case to give a total sample size of 249. Participant screening, inclusion, and exclusion are detailed in Figure 1. The majority of controls did not have available results for free thyroxine $(67 \%$ missing

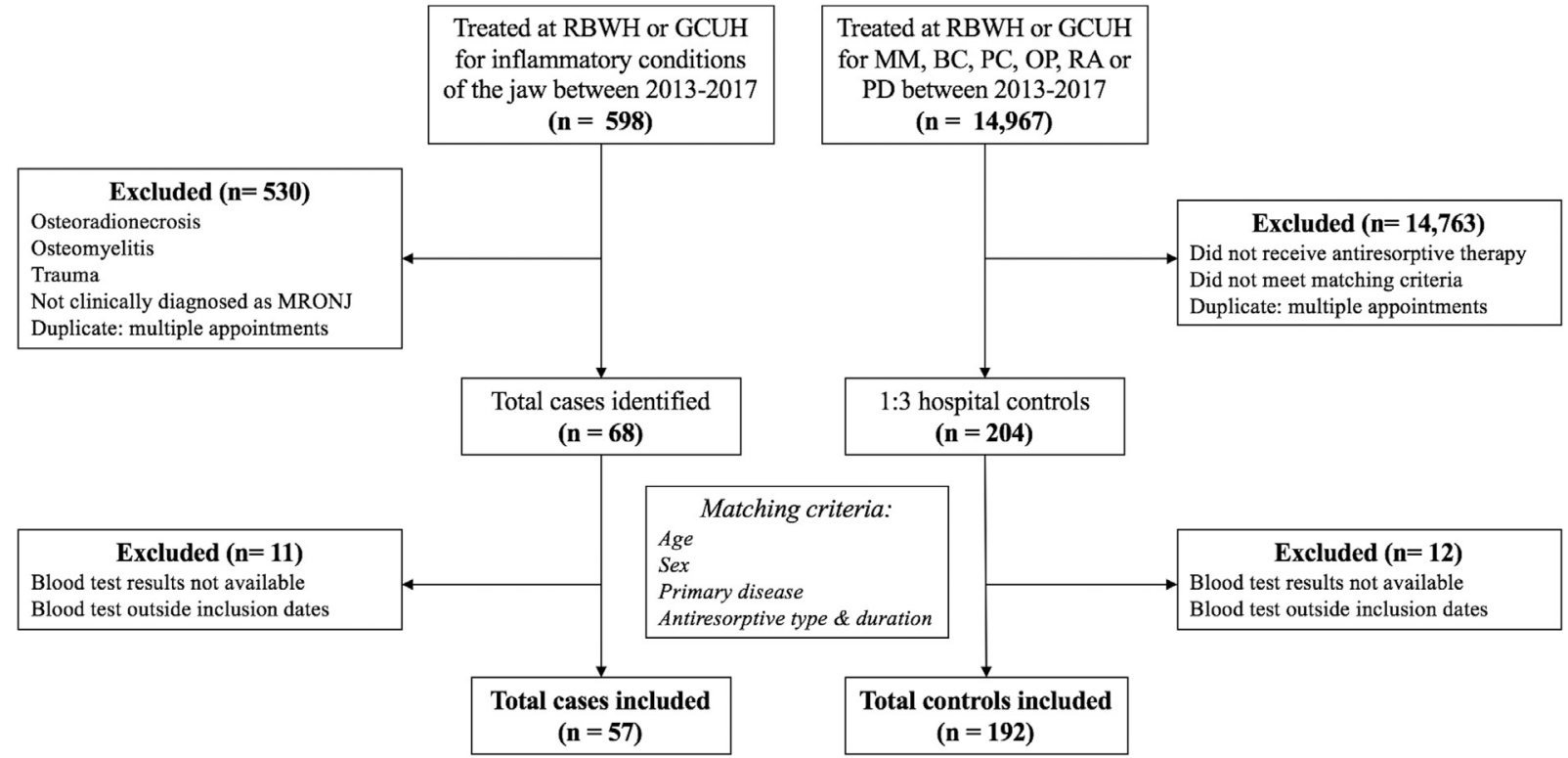

Fig. 1. Participant selection, exclusion, and inclusion of cases and controls using ICD coding data and manual chart reviews. $R B W H$, Royal Brisbane and Women's Hospital; $G C U H$, Gold Coast University Hospital; $M M$, multiple myeloma; $B C$, breast cancer; $O P$, osteoporosis; $R A$, rheumatoid arthritis; $P D$, Paget's disease. 
Table I. Distribution of characteristics and demographic characteristics between cases and controls

\begin{tabular}{|c|c|c|c|}
\hline Characteristics & $\begin{array}{l}\text { Case } \\
(N=57) \\
\mathrm{n}(\%)\end{array}$ & $\begin{array}{l}\text { Control } \\
(N=192) \\
\mathrm{n}(\%)\end{array}$ & $P$ \\
\hline Sex & & & .97 \\
\hline Male & $23(40.4)$ & $78(40.6)$ & \\
\hline Female & 34 (59.6) & $114(59.4)$ & \\
\hline Age & & & .61 \\
\hline $41-60$ & $16(28.1)$ & $42(21.9)$ & \\
\hline $61-80$ & $29(50.9)$ & $103(53.6)$ & \\
\hline $81-100$ & $12(21.1)$ & $47(24.5)$ & \\
\hline Socioeconomic status & & & .67 \\
\hline Low $(1-33)$ & $15(26.3)$ & $42(21.9)$ & \\
\hline Medium (34-66) & $22(38.6)$ & $73(38.0)$ & \\
\hline $\operatorname{High}(67-100)$ & $20(35.1)$ & $77(40.1)$ & \\
\hline Primary disease & & & .61 \\
\hline Osteoporosis & $24(42.1)$ & $81(42.2)$ & \\
\hline Multiple myeloma & $16(28.1)$ & $50(26.0)$ & \\
\hline Breast cancer & $7(12.3)$ & $23(12.0)$ & \\
\hline Prostate cancer & $7(12.3)$ & $28(14.6)$ & \\
\hline Rheumatoid arthritis & $2(3.5)$ & $9(4.7)$ & \\
\hline Paget's disease & $1(3.6)$ & $1(0.5)$ & \\
\hline Antiresorptive therapy & & & .71 \\
\hline Alendronate (Fosamax) & $12(21.1)$ & $35(18.2)$ & \\
\hline Zoledronic acid (Aclasta) & $4(7.0)$ & $13(6.8)$ & \\
\hline Zoledronic acid (Zometa) & $13(22.8)$ & $39(20.3)$ & \\
\hline Pamidronate (Aredia) & $6(10.5)$ & $21(10.9)$ & \\
\hline Risendronate (Actonel) & $7(21.1)$ & $18(18.2)$ & \\
\hline Denosumab (Prolia) & $3(5.3)$ & $13(6.8)$ & \\
\hline Denosumab (Xgeva) & $6(10.5)$ & $33(17.2)$ & \\
\hline Combination & $6(10.5)$ & $20(10.4)$ & \\
\hline $\begin{array}{l}\text { Duration antiresorptive } \\
\text { therapy }\end{array}$ & & & $.003 *$ \\
\hline $1-3 \mathrm{y}$ & $35(67.3)$ & $79(44.1)$ & \\
\hline $4+\mathrm{y}$ & $17(32.7)$ & $100(55.9)$ & \\
\hline Diabetes mellitus & $16(28.1)$ & $25(13.0)$ & $.007 *$ \\
\hline Current smoker & $15(27.8)$ & $21(11.6)$ & .004 \\
\hline Thyroid disease & $7(12.3)$ & $17(8.9)$ & .59 \\
\hline $\begin{array}{l}\text { Autoimmune/inflammatory } \\
\text { disease }\end{array}$ & $15(26.3)$ & $44(22.9)$ & .60 \\
\hline Chemotherapy & & & .95 \\
\hline Oncology & $29(50.9)$ & $102(53.1)$ & \\
\hline Other systemic disease & $2(3.5)$ & $7(3.6)$ & \\
\hline Corticosteroids & & & .29 \\
\hline Inhaled & $5(8.9)$ & $16(8.3)$ & \\
\hline Systemic & $31(55.4)$ & $84(43.8)$ & \\
\hline Kidney disease & $15(26.3)$ & $14(7.3)$ & $<.000 *$ \\
\hline
\end{tabular}

*Significant at $P<0.05$.

data), thyroid-stimulating hormone $(65 \%$ missing data), vitamin D (77\% missing data), CRP (70\% missing data), and erythrocyte sedimentation rate $(82 \%$ missing data), so the analyses were limited to the results of full blood counts. Fasting blood glucose results were available for $68 \%$ of participants, and $11.8 \%$ of cases and $10.8 \%$ of controls reported fasting blood glucose levels outside of the reference range. This difference was not significant in the univariate (OR $0.7 ; 95 \%$ CI: $0.3,2.0 ; P=.54$ ) or multivariate models (OR 0.6; 95\% CI: $0.2,1.6 ; P=.51)$. For this reason, diabetes was not included as a confounder in the multivariable models.

A total of $60 \%$ of participants were female, and 53\% were receiving AR medications for cancer (multiple myeloma, breast cancer, or prostate cancer). The age of participants ranged from 43 to 93 , and the mean \pm standard deviation age of cases was $68.8 \pm 12.6$ years compared with $70.1 \pm 12.1$ years for controls. The average duration of antiresorptive therapy was $3.1 \pm 2.0$ years for cases and $4.1 \pm 2.3$ years for controls. Demographic and clinical information are presented in Table I. Patients who were cases were more likely to be current smokers and have diagnosed diabetes mellitus or kidney disease.

Blood test results are presented in Table II as the frequency (percentage) of cases and controls with parameters outside the laboratory-specific reference range, including stratification as "below" or "above" the range. Mean values are not reported because blood tests were analyzed by 4 different pathology laboratories. At total of $69 \%$ of cases and $65 \%$ of controls reported at least 1 out-of-range result. This was not associated with the risk of developing MRONJ in the univariable (OR 1.1; $95 \% \mathrm{CI}=0.6,2.3 ; P=.71)$ or multivariable $(\mathrm{OR}=0.7$; $95 \% \mathrm{CI}=0.3,1.5 ; P=.29)$ analyses. Cases were more likely to report WBCCs greater than laboratory reference ranges ( $16 \%$ of cases compared with $7 \%$ of controls), and this was significant in the univariable analysis (OR 1.6; 95\% CI $=1.0,2.5 ; P=.03)$ but not in the multivariable model once smoking status, kidney disease, and duration of antiresorptive therapy were adjusted for $(\mathrm{OR}=1.4 ; 95 \% \mathrm{CI}=0.7,2.7 ; P=.39)$. The most commonly reported abnormal result for both groups was hemoglobin, with 53\% of cases and $48 \%$ of controls measuring less than the reference range $(\mathrm{OR}=0.9 ; 95 \% \mathrm{CI}=0.4,2.3 ; P=.86)$.

Table III shows the proportion of oncology and nononcology patients who returned results outside the laboratory-specific reference range. The nononcology subgroup contained 118 patients with osteoporosis (89\%), rheumatoid arthritis (9\%), or Paget's disease (2\%). The oncology subgroup comprised 131 patients with multiple myeloma (50\%), prostate cancer (27\%), or breast cancer $(23 \%)$.

In the nononcology subgroup, $41 \%$ of cases reported WBCCs outside the reference range compared with $14 \%$ of controls. The association between WBCCs and the development of MRONJ was significant in the univariable model $(\mathrm{OR}=2.3 ; 95 \% \mathrm{CI}=1.3,3.9 ; P<.01)$ but not in the multivariable model $(\mathrm{OR}=2.2$; $95 \% \mathrm{CI}=0.9,5.7 ; P=.10$ ) once smoking status, kidney disease, and duration of AR therapy were adjusted for. In the oncology subgroup, $10 \%$ of cases and $17 \%$ of controls reported WBCCs outside of the reference range, and this difference was not significant in either 
Table II. Frequency (percentage) of patients with results outside the laboratory-specific reference range

\begin{tabular}{|c|c|c|c|c|}
\hline Characteristics & $\begin{array}{c}\text { Case }(N=57) \\
n, \%\end{array}$ & $\begin{array}{c}\text { Control }(N=192) \\
n, \%\end{array}$ & $\begin{array}{l}\text { Univariate } \\
\text { OR }(95 \% \text { CI })\end{array}$ & $\begin{array}{c}\text { Multivariate } \\
\text { aOR }(95 \% \text { CI })\end{array}$ \\
\hline$\geq 1$ out-of-range result & $38(69.1)$ & $124(64.6)$ & $1.1(0.6,2.3)$ & $0.7(0.3,1.5)$ \\
\hline Red blood cell count & $12(22.2)$ & $32(16.8)$ & $1.5(0.6,3.4)$ & $0.6(0.2,2.1)$ \\
\hline Below reference range & $12(22.2)$ & $32(16.8)$ & & \\
\hline Above reference range & $0(0)$ & $0(0)$ & & \\
\hline Hematocrit & $18(33.3)$ & $47(24.6)$ & $1.6(0.8,3.4)$ & $0.8(0.3,2.4)$ \\
\hline Below reference range & $18(33.3)$ & $47(24.6)$ & & \\
\hline Above reference range & $0(0)$ & $0(0)$ & & \\
\hline Hemoglobin & $29(52.7)$ & $92(48.2)$ & $1.1(0.6,2.2)$ & $0.9(0.4,2.3)$ \\
\hline Below reference range & $29(52.7)$ & $92(48.2)$ & & \\
\hline Above reference range & $0(0)$ & $0(0)$ & & \\
\hline Mean corpuscular hemoglobin & $1(2.3)$ & $6(3.8)$ & $0.6(0.1,4.7)$ & $0.2(0.0,3.7)$ \\
\hline Below reference range & $1(2.3)$ & $6(3.8)$ & & \\
\hline Above reference range & $0(0)$ & $0(0)$ & & \\
\hline Mean corpuscular volume & $0(0.0)$ & $16(8.3)$ & $0.6(0.0,5.6)$ & - \\
\hline Below reference range & $0(0.0)$ & $10(5.2)$ & & \\
\hline Above reference range & $0(0.0)$ & $6(3.1)$ & & \\
\hline White blood cell count & $13(23.6)$ & $30(15.7)$ & $1.6(1.0,2.5)^{*}$ & $1.4(0.7,2.7)$ \\
\hline Below reference range & $4(7.3)$ & $17(8.9)$ & & \\
\hline Above reference range & $9(16.4)$ & $13(6.8)$ & & \\
\hline Neutrophil count & $14(25.5)$ & $39(20.5)$ & $1.3(0.9,2.0)$ & $1.0(0.5,1.9)$ \\
\hline Below reference range & $7(12.7)$ & $21(11.1)$ & & \\
\hline Above reference range & $9(16.4)$ & $18(9.5)$ & & \\
\hline Lymphocyte count & $15(27.8)$ & $64(33.5)$ & $0.9(0.5,1.7)$ & $0.8(0.3,1.9)$ \\
\hline Below reference range & $13(24.1)$ & $62(32.5)$ & & \\
\hline Above reference range & $2(3.7)$ & $2(1.0)$ & & \\
\hline Monocyte count & $10(17.5)$ & $23(11.0)$ & $1.4(0.9,2.3)$ & $1.2(0.7,2.3)$ \\
\hline Below reference range & $2(3.5)$ & $6(3.1)$ & & \\
\hline Above reference range & $8(14.0)$ & $17(8.9)$ & & \\
\hline Eosinophil count & $3(5.9)$ & $14(7.3)$ & $0.9(0.5,1.8)$ & $0.8(0.3,2.6)$ \\
\hline Below reference range & $0(0.0)$ & $0(0.0)$ & & \\
\hline Above reference range & $3(5.9)$ & $14(7.3)$ & & \\
\hline Basophil count & $0(0.0)$ & $0(0.0)$ & - & - \\
\hline Below reference range & $0(0.0)$ & $0(0.0)$ & & \\
\hline Above reference range & $0(0.0)$ & $0(0.0)$ & & \\
\hline Platelets & $14(24.6)$ & $44(23.7)$ & $1.4(0.7,3.0)$ & $1.3(0.5,3.3)$ \\
\hline Below reference range & $11(19.3)$ & $39(21.1)$ & & \\
\hline Above reference range & $3(5.3)$ & $5(2.6)$ & & \\
\hline
\end{tabular}

$O R$, odds ratio; $C I$, confidence interval; $a O R$, adjusted odds ratio.

Adjusted for current smoking status, duration of antiresorptive therapy, and kidney disease.

*Significant at $P<.05$.

the univariable or multivariable analyses $(\mathrm{OR}=0.6$; $95 \% \mathrm{CI}=0.2,2.2 ; P=.60)$.

In the nononcology subgroup, $44 \%$ of cases had abnormal neutrophil counts compared with $20.5 \%$ of controls. This was associated with increased odds of developing MRONJ (OR $=1.9 ; 95 \% \mathrm{CI}=1.1,3.3$; $P=.018$ ) in the univariate analysis but was no longer significant once smoking status, kidney disease, and duration of AR therapy were adjusted for $(\mathrm{OR}=2.3$; $95 \% \mathrm{CI}=0.8,6.8 ; P=.13)$. In the oncology subgroup, $18.5 \%$ of cases and $25.9 \%$ of controls reported neutrophil counts outside the laboratory reference range, and this difference was not significant in either the univariable or multivariable analyses $(\mathrm{OR}=0.3,95 \%$ $\mathrm{CI}=0.1,1.4 ; P=.13$ ).

\section{DISCUSSION}

Patients who receive AR medications for the treatment of cancer, osteoporosis, and rheumatoid arthritis often return blood tests with parameters outside of standard laboratory reference ranges. The proportion of abnormal results was similar for patients who developed MRONJ and those who did not, and low hemoglobin was the most common out-of-range result. This highlights the importance of a control group when interpreting clinical parameters. One quarter of patients had been diagnosed with an autoimmune disease and more than half of all patients had received chemotherapy and/or corticosteroids. Diabetes, smoking, kidney disease, and thyroid disease were commonly reported by both cases and controls, although more often by cases. With the additional 
Table III. Results outside laboratory reference ranges for oncology and nononcology subgroups

\begin{tabular}{|c|c|c|c|c|c|c|c|c|}
\hline \multirow[b]{2}{*}{ Blood test } & \multicolumn{4}{|c|}{ Oncology } & \multicolumn{4}{|c|}{ Nononcology } \\
\hline & $\begin{array}{c}\text { Case } \\
(N=30) \\
n, \%\end{array}$ & $\begin{array}{c}\text { Control } \\
(N=101) \\
n, \%\end{array}$ & $\begin{array}{c}O R \\
(95 \% C I)\end{array}$ & $\begin{array}{c}a O R \\
(95 \% C I)\end{array}$ & $\begin{array}{c}\text { Case } \\
(N=27) \\
n, \%\end{array}$ & $\begin{array}{c}\text { Control } \\
(N=91) \\
n, \%\end{array}$ & $\begin{array}{c}O R \\
(95 \% C I)\end{array}$ & $\begin{array}{c}a O R \\
(95 \% C I)\end{array}$ \\
\hline$\geq 1$ out-of-range result & $20(66.7)$ & $67(65.7)$ & $0.8(0.3,2.1)$ & $0.8(0.3,2.4)$ & $18(72.0)$ & $57(63.3)$ & $1.7(0.6,4.7)$ & $0.5(0.1,1.9)$ \\
\hline Red blood cell count & $7(24.1)$ & $23(22.8)$ & $1.0(0.3,2.7)$ & $0.2(0.0,1.6)$ & $5(20.0)$ & $9(10.1)$ & $3.5(0.8,15.2)$ & $3.6(0.4,30.2)$ \\
\hline Below reference range & $2(6.7)$ & $13(12.9)$ & & & $2(8.0)$ & $4(4.4)$ & & \\
\hline Above reference range & $1(3.3)$ & $4(4.0)$ & & & $8(32.0)$ & $9(10.0)$ & & \\
\hline Hematocrit & $10(34.5)$ & $32(31.7)$ & $1.0(0.4,2.7)$ & $0.4(0.1,2.1)$ & $8(32.0)$ & $15(16.7)$ & $3.0(0.9,9.8)$ & $2.2(0.5,10.9)$ \\
\hline Below reference range & $2(6.7)$ & $13(12.9)$ & & & $2(8.0)$ & $4(4.4)$ & & \\
\hline Above reference range & $1(3.3)$ & $4(4.0)$ & & & $8(32.0)$ & $9(10.0)$ & & \\
\hline Hemoglobin & $19(63.3)$ & $54(53.5)$ & $1.2(0.5,3.2)$ & $0.7(0.2,2.6)$ & $10(40.0)$ & $38(42.2)$ & $1.0(0.4,2.6)$ & $1.2(0.3,4.5)$ \\
\hline Below reference range & $2(6.7)$ & $13(12.9)$ & & & $2(8.0)$ & $4(4.4)$ & & \\
\hline Above reference range & $1(3.3)$ & $4(4.0)$ & & & $8(32.0)$ & $9(10.0)$ & & \\
\hline Mean corpuscular hemoglobin & $0(0.0)$ & $2(2.6)$ & - & - & $1(4.8)$ & $4(5.1)$ & $0.7(0.1,6.1)$ & $0.5(0.0,7.0)$ \\
\hline Below reference range & $2(6.7)$ & $13(12.9)$ & & & $2(8.0)$ & $4(4.4)$ & & \\
\hline Above reference range & $1(3.3)$ & $4(4.0)$ & & & $8(32.0)$ & $9(10.0)$ & & \\
\hline Mean corpuscular volume & $0(0.0)$ & $5(5.0)$ & $0.1(0.0,77.9)$ & - & $0(0.0)$ & $11(12.1)$ & $0.1(0.0,23.1)$ & - \\
\hline Below reference range & $2(6.7)$ & $13(12.9)$ & & & $2(8.0)$ & $4(4.4)$ & & \\
\hline Above reference range & $1(3.3)$ & $4(4.0)$ & & & $8(32.0)$ & $9(10.0)$ & & \\
\hline White blood cell count & $3(10.0)$ & $17(16.8)$ & $0.7(0.2,1.9)$ & $0.6(0.2,2.2)$ & $10(41.0)$ & $13(14.4)$ & $2.3(1.3,3.9)^{*}$ & $2.2(0.9,5.7)$ \\
\hline Below reference range & $2(6.7)$ & $13(12.9)$ & & & $2(8.0)$ & $4(4.4)$ & & \\
\hline Above reference range & $1(3.3)$ & $4(4.0)$ & & & $8(32.0)$ & $9(10.0)$ & & \\
\hline Neutrophil count & $5(18.5)$ & $21(25.9)$ & $0.6(0.3,1.5)$ & $0.3(0.1,1.4)$ & $11(44.0)$ & $18(20.5)$ & $1.9(1.1,3.3)^{\dagger}$ & $2.3(0.8,6.8)$ \\
\hline Below reference range & $5(18.5)$ & $16(15.7)$ & & & $2(8.0)$ & $5(5.7)$ & & \\
\hline Above reference range & $0(0.0)$ & $5(4.9)$ & & & $9(36.0)$ & $13(14.8)$ & & \\
\hline Lymphocyte count & $8(27.2)$ & $35(34.7)$ & $0.7(0.3,1.9)$ & $0.8(0.2,2.7)$ & $7(28.0)$ & $29(32.2)$ & $1.0(0.4,2.5)$ & $1.0(0.3,3.9)$ \\
\hline Below reference range & $7(24.1)$ & $34(33.7)$ & & & $6(24.0)$ & $28(31.1)$ & & \\
\hline Above reference range & $1(3.4)$ & $1(1.0)$ & & & $1(4.0)$ & $1(1.1)$ & & \\
\hline Monocyte count & $3(10.3)$ & $10(9.9)$ & $1.1(0.5,2.4)$ & $1.1(0.4,2.9)$ & $7(28.0)$ & $13(14.4)$ & $1.7(0.9,3.1)$ & $1.8(0.8,4.2)$ \\
\hline Below reference range & $0(0.0)$ & $4(4.0)$ & & & $2(8.0)$ & $2(2.2)$ & & \\
\hline Above reference range & $3(10.3)$ & $6(5.9)$ & & & $5(20.0)$ & $11(12.2)$ & & \\
\hline Eosinophil count & $0(0.0)$ & $11(10.9)$ & $0.2(0.0,6.2)$ & - & $3(12.5)$ & $3(3.3)$ & $1.7(0.8,3.7)$ & $2.0(0.5,9.0)$ \\
\hline Below reference range & $0(0.0)$ & $0(0.0)$ & & & $0(0.0)$ & $0(0.0)$ & & \\
\hline Above reference range & $0(0.0)$ & $11(10.9)$ & & & $3(12.5)$ & $3(3.3)$ & & \\
\hline Basophil count & $0(0.0)$ & $0(0.0)$ & - & - & $0(0.0)$ & $0(0.0)$ & - & - \\
\hline Below reference range & $0(0.0)$ & $0(0.0)$ & & & $0(0.0)$ & $0(0.0)$ & & \\
\hline Above reference range & $0(0.0)$ & $0(0.0)$ & & & $0(0.0)$ & $0(0.0)$ & & \\
\hline Platelets & $9(31.0)$ & $21(21.4)$ & $1.4(0.5,3.5)$ & $1.8(0.4,7.3)$ & $5(23.8)$ & $23(26.1)$ & $1.5(0.7,3.1)$ & $0.9(0.2,3.9)$ \\
\hline Below reference range & $9(31.0)$ & $19(19.4)$ & & & $2(9.5)$ & $20(22.7)$ & & \\
\hline Above reference range & $0(0.0)$ & $2(2.0)$ & & & $3(14.3)$ & $3(3.4)$ & & \\
\hline
\end{tabular}

$O R$, odds ratio; $C I$, confidence interval; $a O R$, adjusted odds ratio.

Adjusted for current smoking status, duration of antiresorptive therapy, and kidney disease.

*Significant at $P<.01$.

$\dagger$ Significant at $P<.05$.

effects of cancer therapies and antiresorptive medications, there are too many confounders and effect modifiers to identify any clinically relevant risk markers for MRONJ from a full blood count.

These findings agree with previous reports that anemia and blood cell count alterations are common findings in patients with MRONJ. ${ }^{24}$ The proportion of patients with WBCCs greater than and less than the reference range were similar to those reported in a previous study of 212 patients with MRONJ. ${ }^{20}$ One prior study ${ }^{15}$ that compared the nadir WBCC of 8 patients with prostate cancer with MRONJ to 48 controls found that a nadir WBCC $<1000 /$ microliter $(\mu \mathrm{L})$ was a significant predictive factor for MRONJ. Although nadir counts were not available for all patients in this study, the subgroup analysis of oncology patients found that nearly twice as many controls reported WBCCs less than the reference range.

There was more pronounced variation between cases and controls in the subgroup of patients who were prescribed AR medications for diseases other than cancer. One-third of cases in the nononcology subgroup reported total WBCC and neutrophil counts greater than the reference range, which suggests that their immune systems were responding to infection, inflammation, or acute stress in the body. It is not possible to infer what 
role oral disease and inflammation played in producing these high cell counts, but a previous study determined that $89 \%$ of this subgroup developed MRONJ as the result of a known dental precipitator. ${ }^{25}$ These included extractions, acute periodontal infections, and denture trauma. Future studies should prospectively evaluate whether the presence of oral disease alters full blood count results and whether this correlates with the risk of MRONJ. This may assist in better understanding the underlying pathogenesis of MRONJ.

An important strength of this study was the carefully matched control group, which minimized the influence of key confounders on the relationship between blood test results and MRONJ. Matching by primary disease produced an almost identical proportion of cases and controls who received chemotherapy and corticosteroids, and this was particularly important for the interpretation of WBCC. However, a critical limitation of this study is that the number and functionality of macrophages cannot be measured via blood tests, and this immune cell population may play an important role in MRONJ pathogenesis. Macrophages form the second line of defense against microbes from the oral cavity when the mucosa (the first line of defense) is breached. Macrophages and osteoclasts are derived from the same myeloid precursor cell, ${ }^{26}$ and macrophages can fuse to form osteoclasts when stimulated by macrophage colony-stimulating factor and receptor activator of nuclear factor $\kappa \mathrm{B}$ ligand. ${ }^{26}$ This intimate relationship with osteoclasts renders macrophages susceptible to the effects of AR medications. ${ }^{11}$ There is a growing body of evidence to suggest that bone and immune systems share mechanisms, niches, cytokines, and receptors ${ }^{27}$ although the relevance of this to MRONJ remains unclear. Even at a basic level, many of the cellular components of the immune system are derived from hematopoiesis, which occurs predominantly in the bone marrow, ${ }^{27}$ and therefore it is biologically plausible that AR therapies would have some effect on immune function. This is supported by limited evidence that indicates denosumab, ${ }^{28} \mathrm{BPs},{ }^{12,29}$ and antiangiogenics ${ }^{30,31}$ interfere with immune and inflammatory responses, and this may have some clinical relevance to MRONJ that could not be detected from standard laboratory blood tests. Previous studies have also found that BPs impair neutrophil function, ${ }^{32,33}$ and therefore the number of neutrophils in a full blood count may not accurately reflect the capacity of the neutrophil population to respond to invading microbes. Further studies that specifically test neutrophil function, monocyte-macrophage function, immunoglobulin levels, and complement activity are needed to examine the effect of antiresorptive medications on the immune response, and whether this is associated with the development of MRONJ.
Blood tests from 4 different laboratories were included in the present study, and this prevented any analysis of the numerical values returned in the pathology reports. Each laboratory had small variations in their standard references ranges, which mandated the use of "below," "within" or "above" categories for each test. Even though laboratory accreditation and regular on-site audits are mandatory in Australia, there will still be some variation in the accuracy of test results from each laboratory and this may have influenced the findings. The use of hospital controls also results in a patient population that is systemically unwell, and this limits the generalizability of these results.

Patients taking AR medications for oncologic and systemic diseases often return blood test results outside the standard laboratory reference range. Abnormal blood test results were not limited to patients who developed MRONJ and do not appear to be clinically useful in identifying high-risk patients. Standard laboratory tests of circulating blood cells may not be representative of the complex interactions between bone and immune systems in the jawbones, and the findings of this study do not preclude immunologic dysfunction as an important factor in MRONJ pathogenesis. Future research should be aimed at better understanding the innate immune response and in particular the role of macrophages in the development of MRONJ.

\section{REFERENCES}

1. Ruggiero SL, Dodson TB, Fantasia J, et al. American Association of Oral and Maxillofacial Surgeons position paper on medication-related osteonecrosis of the jaw-2014 update. J Oral Maxillofac Surg. 2014;72:1938-1956.

2. Khan AA, Morrison A, Hanley DA, et al. Diagnosis and management of osteonecrosis of the jaw: a systematic review and international consensus. J Bone Miner Res. 2015;30:3-23.

3. Diniz-Freitas M, Fernández-Feijoo J, Diz Dios P, Pousa X, Limeres J. Denosumab-related osteonecrosis of the jaw following non-surgical periodontal therapy: a case report. J Clin Periodontol. 2018;45:570-577

4. Scoletta M, Arata V, Arduino PG, et al. Tooth extractions in intravenous bisphosphonate-treated patients: a refined protocol. J Oral Maxillofac Surg. 2013;71:994-999.

5. Jadu F, Lee L, Pharoah M, Reece D, Wang L. A retrospective study assessing the incidence, risk factors and comorbidities of pamidronate-related necrosis of the jaws in multiple myeloma patients. Ann Oncol. 2007;18:2015-2019.

6. McGowan K, McGowan T, Ivanovski S. Risk factors for medication-related osteonecrosis of the jaws: a systematic review. Oral Dis. 2018;24:527-536.

7. Kalyan S, Chandrasekaran V, Quabius ES, Lindhorst TK, Kabelitz D. Neutrophil uptake of nitrogen-bisphosphonates leads to the suppression of human peripheral blood $\gamma \delta \mathrm{T}$ cells. Cell $\mathrm{Mol}$ Life Sci. 2014;71:2335-2346.

8. Kuiper J, Forster C, Sun C, Peel S, Glogauer M. Zoledronate and pamidronate depress neutrophil functions and survival in mice. Br J Pharmacol. 2012;165:532-539

9. Kalyan S, Quabius ES, Wiltfang J, Mönig H, Kabelitz D. Can peripheral blood $\gamma \delta \mathrm{T}$ cells predict osteonecrosis of the jaw? An 
immunological perspective on the adverse drug effects of aminobisphosphonate therapy. J Bone Miner Res. 2013;28:728-735.

10. Kalyan S, Wang J, Quabius ES, et al. Systemic immunity shapes the oral microbiome and susceptibility to bisphosphonate-associated osteonecrosis of the jaw. J Transl Med. 2015;13:1.

11. Pazianas M. Osteonecrosis of the jaw and the role of macrophages. J Natl Cancer Inst. 2011;103:232-240.

12. Hoefert S, Hoefert CS, Munz A, et al. Effect of bisphosphonates on macrophagic THP-1 cell survival in bisphosphonate-related osteonecrosis of the jaw (BRONJ). Oral Surg Oral Med Oral Pathol Oral Radiol. 2016;121:222-232.

13. Enciso R, Keaton J, Saleh N, Ahmadieh A, Clark GT, Sedghizadeh PP. Assessing the utility of serum C-telopeptide cross-link of type 1 collagen as a predictor of bisphosphonate-related osteonecrosis of the jaw: a systematic review and meta-analysis. $J$ Am Dent Assoc. 2016.

14. Dal Prá K, Lemos C, Okamoto R, Soubhia A, Pellizzer E. Efficacy of the C-terminal telopeptide test in predicting the development of bisphosphonate-related osteonecrosis of the jaw: a systematic review. Int J Oral Maxillofac Surgs. 2017;46:151-156.

15. Miyazaki H, Nishimatsu H, Kume H, et al. Leukopenia as a risk factor for osteonecrosis of the jaw in metastatic prostate cancer treated using zoledronic acid and docetaxel. BJU Int. 2012;110:E520-E525.

16. Fehm T, Beck V, Banys M, et al. Bisphosphonate-induced osteonecrosis of the jaw (ONJ): incidence and risk factors in patients with breast cancer and gynecological malignancies. Gynecol Oncol. 2009;112:605-609.

17. Matsui A, Kurihara J, Morishima H, et al. Medication related osteonecrosis of the jaw (MRONJ): a retrospective survey of a series of patients treated according to the AAOMS guidelines. $J$ Oral Maxillofac Surg Med Pathol. 2015;27:757-763.

18. Hayashida S, Soutome S, Yanamoto S, et al. Evaluation of the treatment strategies for medication-related osteonecrosis of the jaws (MRONJ) and the factors affecting treatment outcome: a multicenter retrospective study with propensity score matching analysis. J Bone Miner Res. 2017;32:2022-2029.

19. Watters AL, Hansen HJ, Williams T, et al. Intravenous bisphosphonate-related osteonecrosis of the jaw: long-term follow-up of 109 patients. Oral Surg Oral Med Oral Pathol Oral Radiol. 2013;115:192-200.

20. Mücke T, Jung M, Mitchell DA, et al. Do measurements of inflammatory mediators in blood predict recurrence in patients with bisphosphonate-related osteonecrosis of the jaws? $\mathrm{Br} J$ Oral Maxillofac Surg. 2016;54:286-289.

21. Akashi M, Wanifuchi S, Iwata E, et al. Differences between osteoradionecrosis and medication-related osteonecrosis of the jaw. Oral Maxillofac Surg. 2018;22:59-63.

22. Von Elm E, Altman DG, Egger M, et al. The Strengthening the Reporting of Observational Studies in Epidemiology (STROBE) statement: guidelines for reporting observational studies. Prev Med. 2007;45:247-251.

23. Australian Bureau of Statistics. Census of population and housing: Socio-economic indexes for areas (SEIFA), Australia, 2016. Canberra, ACT, Australia: Australian Bureau of Statistics; 2018. Available from: http://www.abs.gov.au/ausstats/abs@.nsf/mf/ 2033.0.55.001. Accessed July 29, 2018.

24. Pires F, Miranda A, Cardoso E, et al. Oral avascular bone necrosis associated with chemotherapy and biphosphonate therapy. Oral Dis. 2005;11:365-369.

25. McGowan K, Ivanovski S, Acton C. Osteonecrosis of the jaws: a 14-year retrospective survey of hospital admissions. Aus Dent J. 2018;63:202-207.

26. Long CL, Humphrey MB. Osteoimmunology: the expanding role of immunoreceptors in osteoclasts and bone remodeling. Bonekey Rep. 2012;1(4).

27. Anthony BA, Link DC. Regulation of hematopoietic stem cells by bone marrow stromal cells. Trends Immunol. 2014;35:32-37.

28. Ferrari-Lacraz S, Ferrari S. Do RANKL inhibitors (denosumab) affect inflammation and immunity? Osteoporos Int. 2011;22:435-446.

29. Hoefert S, Hoefert CS, Munz A, et al. Altered macrophagic THP-1 cell phagocytosis and migration in bisphosphonaterelated osteonecrosis of the jaw (BRONJ). Clin Oral Investig. 2016;20:1043-1054.

30. Mashkani B, Griffith R, Ashman LK. Colony stimulating factor1 receptor as a target for small molecule inhibitors. Bioorganic Med Chem. 2010;18:1789-1797.

31. Terme M, Colussi O, Marcheteau E, Tanchot C, Tartour E, Taieb J. Modulation of immunity by antiangiogenic molecules in cancer. Clin Dev Immunol. 2012;2012.

32. Favot CL, Forster C, Glogauer M. The effect of bisphosphonate therapy on neutrophil function: a potential biomarker. Int J Oral Maxillofac Surg. 2013;42:619-626.

33. Hagelauer N, Pabst AM, Ziebart T, Ulbrich H, Walter C. In vitro effects of bisphosphonates on chemotaxis, phagocytosis, and oxidative burst of neutrophil granulocytes. Clin Oral Invest. 2015;19:139-148.

\section{Reprint Requests:}

\section{Kelly McGowan}

Mailbox 22

Oral Health Centre

288 Herston Road

Herston QLD 4006

Australia.

K.mcgowan@uq.edu.au 